\title{
Competencia digital y planificación curricular en docentes de centros de educación básica alternativa. Ica, 2021
}

\section{RESUMEN}

Miryam Gladys Pineda Morán miryamgladys4164@gmail.com https://orcid.org/0000-0002-2307-9191

Nicanor Piter Saavedra Carrión nicanor.saavedra@ayacucho.edu.pe https://orcid.org/0000-0001-5827-9524

Gabriela Soledad Vásquez Urbina gvasquezur@ucvvirtual.edu.pe https://orcid.org/0000-0002-1286-344X

Cindy Vílchez Ramírez vilchez.cindy@gmail.com https://orcid.org/0000-0001-7988-8580 Universidad César Vallejos

El presente estudio tuvo como objetivo determinar la influencia de la competencia digital en la planificación curricular en docentes de los CEBA del departamento de Ica, de tipo aplicado, de enfoque cuantitativo, de método hipotético deductivo, de nivel explicativo y de diseño no experimental, correlacional causal, de corte transeccional, el cual contó con un universo poblacional de 214 docentes que laboran en diversos CEBA de ciclo avanzado del departamento de Ica, y por muestreo probabilístico estratificado, se determinó la muestra la cual estuvo integrada por 138 docentes, a quienes se les aplicó dos cuestionarios para recabar la información de las variables, los cuales fueron validados por expertos y determinado su confiabilidad por el coeficiente de Cronbach. En relación a los resultados, se pudo afirmar que la significancia del informe de ajuste de modelo fue inferior al margen de error, por tanto se rechazó la hipótesis nula, aseverando que la competencia digital es factor predictor de la planificación curricular, asimismo, según la prueba de Pseudo R2, se pudo concluir que la competencia digital explica según Nagelkerke el 40,3 \% de la planificación curricular y para Cox y Snell el 19,4 \%, es decir, que el desarrollo óptimo de la competencia digital provoca efecto positivo sobre la planificación curricular.

Palabras clave: competencia digital; planificación curricular; desarrollo profesional docente. 


\title{
Digital competence and curricular planning in teachers of alternative basic education centers. Ica, 2021
}

\begin{abstract}
The objective of this study was to determine the influence of digital competence in curriculum planning in teachers of the CEBA of the department of Ica, applied, quantitative approach, hypothetical deductive method, explanatory level and nonexperimental design, causal correlational, cross-sectional cut, which had a population universe of 214 teachers who work in various advanced cycle CEBAs in the department of Ica, and by stratified probability sampling, the sample was determined which was composed of 138 teachers, to whom two questionnaires were applied to collect information on the variables, which were validated by experts and their reliability was determined by Cronbach's coefficient. In relation to the results, it was possible to affirm that the significance of the model adjustment report was lower than the margin of error, therefore the null hypothesis was rejected, asserting that digital competence is a predictor of curriculum planning, also, according to the Pseudo R2 test, it could be concluded that digital competence explains according to Nagelkerke $40.3 \%$ of curriculum planning and for Cox and Snell 19.4\%, that is, that the optimal development of digital competence causes positive effect on curriculum planning.
\end{abstract}

Keywords: digital competence; curriculum planning; teacher professional development.

Artículo recibido: 20 diciembre. 2021

Aceptado para publicación: 10 enero 2022

Correspondencia: miryamgladys4164@gmail.com

Conflictos de Interés: Ninguna que declarar 


\section{INTRODUCCIÓN}

\section{Planteamiento del problema}

Desde la perspectiva del personal directivo de una escuela nacional, es relevante y necesario que su conducción priorice el proceso de enseñanza, reforzando la planificación curricular conjuntamente con los facilitadores del saber que son los mismos docentes, creando actividades, empoderándolos de estrategias, recopilando y analizando evidencias sobre el avance escolar, además del reajuste de los aprendizajes a alcanzar por grado, traduciéndolo en la inserción para la mejora de los aprendizajes, cabe señalar que aunque todo lo anterior es cierto, el personal directivo no solo enfrenta de manera directa al proceso de enseñanza en la escuela, sino también a nivel de planificación curricular (Donoso y Benavides, 2018).

Asimismo, en la actualidad, las personas han ido incorporando a su quehacer cotidiano una gran cantidad de herramientas tecnológicas, además, el uso de internet ha renovado la concepción sobre la palabra digitalizar y ha empoderado a la información dinamizando el proceso y manejo del conocimiento. Ello ha llegado a repercutir en la sociedad y sin duda ha llevado a cambiar la perspectiva que se llevaba hasta el momento (Lévano et ál., 2019). Sobre las competencias digitales, Almerich et ál. (2018), nos expresó que verdaderamente sus efectos del empoderamiento de dichas competencias serán trascendentes y surtirán efectos positivos al ser integradas en nuestras actividades pedagógicas, enriqueciendo la planificación curricular, convirtiéndolo en documento fortalecido y vivos.

Cabe considerar, que según la UNESCO (2013), a través de sus formuladores de políticas educativas, buscan que países aliados integren mediante el uso de TIC una educación inclusiva, con el fin de conectar las escuelas a programas actuales y reformas en educación dependiendo de las necesidades que muestre cada país. En Ecuador, un factor esencial latente en los docentes que causa la deficiente adquisición de competencias digitales en el actual siglo XXI, llega a corresponder al bajo uso de los recursos tecnológicos, que tienen las instituciones educativas, siendo el responsable inmediato el gobierno estatal, en otras palabras García et ál. (2016), expresó que a pesar de tener conocimiento sobre el manejo adecuado de recursos tecnológicos, existen docentes que se rehúsan aplicarlas en su labor pedagógica; siendo una causa más para que no se aprovechen las competencias digitales de forma eficiente. 
Como se ha afirmado, la competencia digital en el mundo es tomada como herramienta útil que fomenta la obtención de destrezas, cualidades y capacidades, cuyo propósito es posibilitar la transferencia de conocimientos cambiando el paradigma tradicional generando innovación (Marza y Cruz, 2018), además, según el Ministerio de Educación Finlandés durante el año 2015, planteó en su currículo la denominada alfabetización digital, desde la primaria, solicitando a los docentes que los estudiantes deben de aprender antes de lograr digitar, argumentando que en el mundo se encuentra a un solo clic de distancia. Francia en el año 2018, prohíbe el uso de dispositivos móviles en sus centros de estudio, ya que son instrumentos innecesarios que generan distracción en los educandos. Asimismo, la UNESCO (2020), considera al docente como mediador del conocimiento indispensable, al ser ellos los que se relacionan formidablemente con los estudiantes, son más cercanos a ellos, son los responsables de su formación.

En el territorio nacional, el MINEDU (2016), indicó que se ha venido experimentando con diferentes recursos tecnológicos y poniendo a prueba prácticas pedagógicas dando una introducción a los estudiantes sobre el uso de recursos digitales, además el MINEDU (2017a), señaló que la palabra planificar es sinónimo de realizar una hipótesis de la labor pedagógica, no es rígida, es el reflejo de un periodo de realización, siendo su variación viable al momento de ser ejecutado, con el propósito de ser pertinente y eficiente.

Además, la implementación de la estrategia Aprendo en Casa (MINEDU, 2020), ha desnudado una gran deficiencia respecto al uso de instrumentos y recursos tecnológicos, siendo la quinta parte del total de docentes, los que no poseen o no han desarrollado competencias digitales, asimismo, Martínez-Garcés (2020), expresaron que la realidad actual es muy incierta, al ser la virtualidad una modalidad de enseñanza obligatoria remota, por ello, todo docente debe capacitarse para afrontar este gran reto y buscar la integración de nuevos métodos de enseñanza integrándolas en sus programaciones curriculares.

Esta realidad la comparten los docentes de los CEBA del departamento de Ica, los cuales atienden a jóvenes, adultos y adultos mayores que por diferentes motivos dejaron de estudiar y que ahora toman la decisión de culminar sus estudios básicos, personas que en su mayoría son trabajadores de las agroexportadores, son moto taxistas, empleados en centros comerciales, pescadores artesanales, trabajadores de la minería, etc., que necesitan que la enseñanza, se adecúe al contexto actual incorporando la educación 
remota para darle continuidad a sus estudios y opten por permanecer y culminarlos; allí la figura docente debe asumir el reto de moderar la inserción de las TIC, de manera ética y didáctica mediante su labor pedagógica, viéndose ello, reflejado en la inserción de los documentos de planificación a largo, mediano y a corto plazo, cabe señalar que la elaboración de dichos documentos resulta muy complicado en la actualidad, a causa de la diversidad de estudiantes que son parte de los CEBA, considerar todas sus características insertarlas en la planificación curricular requiere de mucha rigurosidad y un trabajo fuerte de equipo.

Por ende, se planteó como problema general: ¿Cómo influye la competencia digital en la planificación curricular en docentes de los CEBA de ciclo avanzado. Ica, 2021?, también como objetivo general: Determinar la influencia de la competencia digital en la planificación curricular en docentes de los CEBA de ciclo avanzado. Ica 2021, y por último como hipótesis: La competencia digital influye significativamente en la planificación curricular en docentes de los CEBA de ciclo avanzado. Ica, 2021.

\section{Marco teórico}

Respecto a la teoría sobre competencia digital, según Idrovo-Ortiz (2019), la principal que aspira declarar el uso de las TIC en el sector educación es el conectivismo, considerado como una corriente pedagógica, el cual busca dar fundamento de forma epistemológica a un novedoso método de enseñanza con el apoyo de las TIC y las redes comunicacionales que en la actualidad son más exigentes para el profesorado. Además, Siemens (2004), declaró que el conectivismo se fundamentó al tomar en cuenta principios diversos de la teoría del caos, de las redes, de la complejidad y de la autoorganización, en tal sentido, la formación sucede en contextos considerados difusos y cambiantes.

Además, el conectivismo de Siemens (2004), enfatizó el uso de herramientas versátiles y competentes al contexto del estudiante, con el propósito de facilitar el conocimiento por medio de clases interactivas apoyadas por las TIC, además, propone la conexión de fuentes de información, para extraer conocimiento de gran relevancia, útil y aplicable. Cabe señalar que ese conocimiento además de describir el presente, debe apoyar en la construcción de un futuro, y para que ello se realice tanto las conexiones y redes deben de proveer información valiosa para tomar decisiones más coherentes, es así, que el conectivismo, incentiva el aprenderaprender por medio del uso de las TIC.

Para el MINEDU (2017b), la competencia es comprendida como la facultad que posee 
una persona al amalgamar una serie de capacidades con el objetivo de alcanzar un propósito específico en una situación cotidiana determinada, conduciendo de forma pertinente y con sentido ético. Asimismo, Pompa y Pérez (2015), definieron a la competencia como todos los comportamientos instruidos por habilidades cognitivas, práctica de valores, destrezas y gran variedad de información, que posibilita resolver de forma eficiente y eficaz cualquier situación que se presente.

Cabe señalar que ser competente, se refiere a comprender el problema que se está afrontando, evaluar las posibilidades que se tengan para darle solución, es decir, que se debe identificar el conocimiento y la habilidad que uno mismo posee o que se encuentran a la mano en el contexto, analizar el posible procedimiento a optar que se amolde a la situación y al propósito, para posteriormente tomar una decisión y de esa manera llevar a cabo el procedimiento que se seleccione (MINEDU, 2017b).

Acto seguido, Almerich et ál. (2018), definieron la competencia digital como un conjunto de destrezas, habilidades y conocimiento adquirido por las personas, los cuales permiten manejar variados recursos tecnológicos. Aportando a lo dicho, Vargas-Murillo (2019), lo definió como la suma de conocimiento, habilidad y actitud, claves para el docente, cuyo propósito es el de continuar aprendiendo permanentemente y desarrollando su profesionalidad que hacen posible el uso de la tecnología de manera crítica y responsable, asimismo, estas competencias que son adquiridas por el docente están estrechamente relacionados con aspectos tecnológicos, informativos, multimedia y comunicativos.

Cabe señalar que la propuesta de dimensiones que se tomó en cuenta está basada en el Marco Común de Competencia Digital Docente (MCCDD), adaptado del Marco europeo sobre competencia digital ciudadana (DigComp), publicado en el 2013, descrito en la INTEF (2017), el cual presenta cinco dimensiones:

Como primera dimensión a la información y alfabetización informacional, la cual consiste en la búsqueda de información, de datos y de contenidos digitalizados en la web, además de la manera de reunir dicha información, procesarla, comprenderla y evaluarla de manera crítica, que conlleve a su almacenamiento para hacer más fácil la recuperación de los mismos siendo organizados adecuadamente para su posterior uso (Vargas-Murillo, 2019). Como segunda dimensión se consideró a la comunicación y colaboración, que consiste en la interacción haciendo uso de entornos digitales, del estar dispuesto a compartir conocimiento y contenido digital, de conectarse y colaborar con otras personas por medio 
de comunidades digitalizadas, cabe resaltar que se debe de respetar las normas de conductas virtual, estando consiente a la diversidad cultural, desarrollando posibles estrategias que contrarresten conductas inadecuadas, y que se cree, adapte y gestione su identidad digital (Vargas-Murillo, 2019). Como tercera dimensión, se contempló a la creación de contenidos digitales, basado en la producción y edición de información digital nueva., es considerada como el proceso de integración y revaloración de conocimiento y contenido formado con anterioridad, respetando la propiedad intelectual (Vargas-Murillo, 2019). La cuarta dimensión es la seguridad, referida a la protección de información relevante y datos personales del usuario: su identidad digital y sus recursos digitales; implementando medidas seguras y haciendo uso responsable de sus dispositivos digitales (Vargas-Murillo, 2019). Y como quinta dimensión a la resolución de problemas, que consiste en la identificación de posibles necesidades al momento de hacer uso de recursos digitales, al tomar decisiones informadas acerca de herramientas tecnológicas más idóneas dependiendo de la necesidad que se tenga para la resolución de problemas conceptuales por medio de entornos digitales, asimismo, se refiere al uso de herramientas digitales con creatividad, resolución de posibles problemas técnicos y la actualización competencial propia y la de otros (Vargas-Murillo, 2019).

Sobre la variable planificación curricular Aza y Lica (2019), la definieron como un proceso organizado de acciones que deben de realizarse en las escuelas con el propósito optimizar el conocimiento por medio de experiencias de aprendizaje acorde con el contexto de los educandos. Esta planificación debe de responder a las metas generales planteadas por el Minedu, cuyo fin radica en su labor orientadora, en donde el profesor puede ordenar lo que pretende abordar en las sesiones de aprendizaje anticipando sucesos y evaluando resultados. Navarro et ál. (2010), lo expresaron como "el proceso mediante el cual se logran los objetivos fijados en una organización a través del cumplimiento de planes, previamente elaborados en un tiempo determinado" (p. 210).

De acuerdo con el MINEDU (2017a), el arte de planificar, configurar y elaborar variados escenarios educativos acordes con el contexto del estudiante y cuyo propósito es el de consolidar los aprendizajes impartidos se le conoce como planificación curricular, también percibida como una hipótesis de la labor pedagógica, basada en un diagnóstico referente a lo que necesitan aprender los estudiantes, a cómo se realizará su ejecución. Cabe mencionar que es posible realizar cambios acordes con las evaluaciones que se 
planteen, con el propósito de que está sea pertinente y eficiente al propósito de aprendizaje ya establecido con anterioridad.

La propuesta de Lozano (2018), consideró al proceso de planificación curricular como un proceso dinamizado, flexible y cambiante, el cual no se percibe como algo fijo e inflexible. Por tal motivo, en dicho proceso se diferencia cuatro fases, que el presente estudio lo toma como sus dimensiones al estar alineado con los objetivos propuestos y que, además, por la actual coyuntura se percibe en el actuar de los docentes.

La evaluación inicial, análisis previo o diagnóstico, es el punto de partida a la hora de realizar la planificación del trabajo pedagógico, ésta fase es de suma importancia porque lo primero que se hace es partir de una evaluación inicial, además de analizar factores socio-educativos, socio-afectivos, los niveles de logro del año anterior, otros, que van a dar a conocer particularidades necesarias que serán soporte de la labor docente (Lozano, 2018), asimismo, Flores (2006), señaló que el diagnóstico tiene como objetivo el análisis real de la educación, permitiendo la obtención de una visión más clara respecto al proceso de enseñanzaaprendizaje, a los propios estudiantes, y a otros procesos que pueden intervenir, como los docentes, materiales o factores del exterior. Según Arriaga (2016), el diagnóstico es considerado como un proceso, descriptivo, clasificatorio, predictivo y explicativo sobre el comportamiento de personas dentro de un sistema escolar, las cuales incluyen en su accionar un sinnúmero de actividades de medición y de evaluación.

La programación curricular, es la segunda fase que se da una vez ya concluido el estudio de la realidad, las variadas posibilidades de acción, establecimiento de priorización curricular y de las necesidades de formación, cabe señalar que luego de ello se estará en condiciones de plantearse objetivos curriculares y experiencias de aprendizaje, ya definido todo ello según Lozano (2018), se inició la construcción de la programación curricular, el cual deberá de establecer un método concreto, organizado tomando en cuenta los diferentes recursos con que se cuente. Flores (2006), da a entender que esta etapa considera la planificación anual, experiencias de aprendizaje y unidades de aprendizaje, en los cuales se consigna todos aquellos aspectos que hacen posible un buen desarrollo del trabajo pedagógico cuya consecuencia de su aplicación, logre alcanzar los objetivos y metas propuestas.

La ejecución curricular, es la etapa de puesta en marcha de la programación curricular, actuando siempre tomando en consideración lo ya planificado. Cabe señalar que durante 
la ejecución es normal que se tomen decisiones dado algunos imprevistos, dificultades, entre otros, que surgen y obligan a realizar algunas modificaciones en la programación (Lozano, 2018), Según Flores (2006), esta etapa es caracterizada por desarrollar experiencias curriculares en interacción docente-estudiante, la cual involucra un papel predominante del profesor respecto al desarrollo de su área curricular. Navarro y Samón (2017), señalaron que, en esta etapa de ejecución, el docente considera acciones y métodos de forma intencionada buscando la construcción del aprendizaje y empoderamiento de competencias reforzando el proceso de enseñanza-aprendizaje, además de utilizar de manera efectiva recursos y materiales didácticos.

La evaluación, es la etapa la cual permitirá que el docente compruebe si realmente está logrando los objetivos que se han planteado (Lozano, 2018). Para Flores (2006), esta etapa involucra la recolección de información sobre el proceder y de las fases que se han considerado en la programación curricular, su finalidad es la de verificar el desarrollo de la programación curricular, de su formulación, su juicio de valor que permiten tomar decisiones oportuna y coherentemente. El MINEDU (2016), señaló que los estándares de aprendizaje, facultan la identificación del avance respecto al logro de sus aprendizajes que se espera que obtengan al finalizar el grado, ciclo o nivel escolar de estudio, además son considerados referentes de evaluación.

\section{ESTRATEGIAS METODOLÓGICAS}

\section{Metodología}

El presente estudio fue de tipo aplicado, de enfoque cuantitativo, de método hipotético deductivo, de nivel explicativo y de diseño no experimental, correlacional causal de corte transeccional.

Figura 1. Diseño de investigación

$$
\mathbf{X}_{1} \rightarrow \mathbf{X}_{2}
$$

Donde, $\mathrm{X}_{1}$ es la variable independiente (competencia digital), $\mathrm{X}_{2}$ es la variable dependiente (planificación curricular) y es la influencia de la variable independiente sobre la dependiente.

\section{Población y muestra}

La población del presente estudio estuvo formada por 214 docentes que laboran en los CEBA de ciclo avanzado de la DRE Ica durante el año 2021. Al respecto, Hernández y Mendoza (2018), señalaron que la población es percibida como un conjunto de personas que se encuentran en un determinado lugar geográfico, claramente definido y que 
contienes características similares.

Tabla 1. Población de estudio

\begin{tabular}{|c|c|c|}
\hline UGEL & CEBA & N. ${ }^{\circ}$ docentes \\
\hline \multirow{7}{*}{ ICA } & San Luis Gonzaga & 11 \\
\hline & Margarita Santa Ana de Benavides & 14 \\
\hline & Fe y Alegría & 8 \\
\hline & Víctor Manuel Maurtua & 16 \\
\hline & Sebastián Barranca & 7 \\
\hline & Juan Pablo Fernandini & 8 \\
\hline & Nuestra Señora de las Mercedes & 13 \\
\hline \multirow{2}{*}{ PALPA } & Raúl Porras Barrenechea & 7 \\
\hline & Palpa & 13 \\
\hline \multirow{4}{*}{ NASCA } & Fermín Del Castillo Arias & 11 \\
\hline & Josefina Mejía de Bocanegra & 12 \\
\hline & Abraham Valdelomar & 11 \\
\hline & San Juan & 10 \\
\hline \multirow{4}{*}{ PISCO } & Banderas del Perú & 5 \\
\hline & José Carlos Mariátegui & 9 \\
\hline & Renán Elías Olivera & 8 \\
\hline & Augusto Salazar Bondi & 5 \\
\hline \multirow{5}{*}{ CHINCHA } & Jhon F. Kennedy & 11 \\
\hline & Rubén Martínez Puicón & 12 \\
\hline & Santa Ana & 7 \\
\hline & Andrés Avelino Cáceres & 7 \\
\hline & Tony Gubbels & 4 \\
\hline TOTAL & & 214 \\
\hline
\end{tabular}

Sobre la muestra, se ha considerado, hacer uso del muestreo probabilístico estratificado, entablando en primer lugar, el tamaño de la muestra haciendo uso de la fórmula finita, la cual determinó que la muestra estaría integrada por 138 docentes, luego se halló el factor de proporción para mantener la representatividad de cada CEBA, por ende al dividir el tamaño de la muestra (138), con el tamaño poblacional (214), se obtiene 0,645 como resultado el cual es multiplicado por la cantidad de docentes de cada CEBA y de esa manera se obtuvo la muestra.

Tabla 2. Muestra de estudio

\begin{tabular}{clccc}
\hline UGEL & \multicolumn{1}{c}{ CEBA } & Población & Factor & Muestra \\
\hline \multirow{2}{*}{ ICA } & San Luis Gonzaga & 11 & 0.645 & 7 \\
& Margarita Santa Ana de Benavides & 14 & 0.645 & 9
\end{tabular}




\begin{tabular}{llccc} 
& Fe y Alegría & 8 & 0.645 & 5 \\
& Víctor Manuel Maurtua & 16 & 0.645 & 11 \\
& Sebastián Barranca & 7 & 0.645 & 5 \\
& Juan Pablo Fernandini & 8 & 0.645 & 5 \\
& Nuestra Señora de las Mercedes & 13 & 0.645 & 9 \\
\hline \multirow{2}{*}{ PALPA } & Raúl Porras Barrenechea & 7 & 0.645 & 5 \\
& Palpa & 13 & 0.645 & 9 \\
\hline \multirow{5}{*}{ NASCA } & Fermín Del Castillo Arias & 11 & 0.645 & 7 \\
& Josefina Mejía de Bocanegra & 12 & 0.645 & 8 \\
& Abraham Valdelomar & 11 & 0.645 & 7 \\
& San Juan & 10 & 0.645 & 6 \\
\hline \multirow{3}{*}{ PISCO } & Banderas del Perú & 5 & 0.645 & 3 \\
& José Carlos Mariátegui & 9 & 0.645 & 6 \\
& Renán Elías Olivera & 8 & 0.645 & 5 \\
& Augusto Salazar Bondi & 5 & 0.645 & 3 \\
\hline \multirow{5}{*}{ CHINCHA A } & Santa Ana & 11 & 0.645 & 7 \\
& Jhon F. Kennedy & 12 & 0.645 & 8 \\
& Andrés Avelino Cáceres & 7 & 0.645 & 5 \\
\hline Total & Tony Gubbels & 7 & 0.645 & 5 \\
\hline \multirow{3}{*}{} & & 4 & 0.645 & 3 \\
\hline & & 214 & & 138 \\
\hline
\end{tabular}

\section{Técnicas e instrumentos}

En relación a la técnica, se utilizó a la encuesta, debido a que, fue un medio sencillo que facilito la recolección de la información de ambas variables de estudio, que fueron relevantes e imprescindibles para apoyar a la corroboración de los supuestos propuestos. Para Carhuancho et al. (2019), la encuesta es considerada como una técnica que se diferencia por su fácil práctica, asimismo, es de aplicación transeccional, en donde la investigación anticipadamente concreta el día y fija la hora para su aplicación a los participantes de la investigación.

Respecto a los instrumentos, se ha utilizado dos cuestionarios, los cuales fueron validados por expertos y con apoyo del alfa de Cronbach, se evidenció su grado de confiabilidad, donde para la competencia digital fue de 0,962 y para la planificación curricular de 0,975; siendo ambos altamente confiables. Al respecto Carrasco (2017), mencionó que el cuestionario permite obtener directamente, y por medio de una hoja de respuestas los datos que se pretenden recolectas, además, la pregunta estandarizada es preparada anticipadamente y con vasta previsión. 


\section{RESULTADOS Y DISCUSIÓN}

\section{Resultados descriptivos}

Tabla 3. Cruce entre la competencia digital y planificación curricular

\begin{tabular}{|c|c|c|c|c|c|c|}
\hline & \multicolumn{3}{|c|}{ Planificación curricular } & \multirow{2}{*}{ Total } \\
\hline & & & Bajo & Medio & Alto & \\
\hline \multirow{6}{*}{$\begin{array}{l}\text { Competencia } \\
\text { digital }\end{array}$} & \multirow{2}{*}{ Básico } & Recuento & 0 & 0 & 0 & 0 \\
\hline & & $\%$ del total & $0,0 \%$ & $0,0 \%$ & $0,0 \%$ & $0,0 \%$ \\
\hline & \multirow{2}{*}{ Intermedio } & Recuento & 0 & 13 & 26 & 39 \\
\hline & & $\%$ del total & $0,0 \%$ & $9,4 \%$ & $18,8 \%$ & $28,3 \%$ \\
\hline & \multirow{2}{*}{ Avanzado } & Recuento & 0 & 1 & 98 & 99 \\
\hline & & $\%$ del total & $0,0 \%$ & $0,7 \%$ & $71,0 \%$ & $71,7 \%$ \\
\hline \multirow{2}{*}{ Total } & & Recuento & 0 & 14 & 124 & 138 \\
\hline & & $\%$ del total & $0,0 \%$ & $10,1 \%$ & $89,9 \%$ & $100,0 \%$ \\
\hline
\end{tabular}

Interpretación: Sobre la competencia digital y planificación curricular tomado de una muestra de 138 docentes, demostró que cuando la competencia digital es considerada en un nivel avanzado, el 71,0\% señaló tener un nivel alto de planificación curricular y el $0,7 \%$ un nivel medio, y cuando la competencia digital es considerada en nivel intermedio, el 18,8\% evalúo a la planificación curricular en alto y el 9,4\% en medio.

\section{Resultados inferenciales}

Tabla 4. Prueba de normalidad

\begin{tabular}{lcccc}
\hline \multicolumn{1}{c}{ Dimensiones y variables } & \multicolumn{3}{c}{ Kolmogorov-Smirnov $^{\mathbf{a}}$} \\
\cline { 2 - 4 } & Estadístico & gl & Sig. \\
\hline Competencia digital &, 114 & 138 &, 000 \\
Planificación curricular &, 152 & 138 &, 000 \\
\hline
\end{tabular}

Interpretación: En la tabla se puede observar que la prueba de K-S ha permitido afirmar que el valor de la significancia de ambas variables fue inferior al margen de error, por ende, se concluyó que no poseen distribución normal, por ende, la prueba de hipótesis se ha realizado con apoyo de la regresión logística ordinal.

Tabla 5. Informe de ajuste de modelo

\begin{tabular}{lcccc}
\hline \multicolumn{1}{c}{ Modelo } & Logaritmo de la verosimilitud -2 & Chi-cuadrado & gl & Sig. \\
\hline Sólo intersección & 35,773 & & & \\
Final & 6,002 & 29,770 & 1 &, 000
\end{tabular}

Función de enlace: Logit. 
Interpretación: Respecto al ajuste de datos evidenciados en la tabla 5, el cual busca encontrar la dependencia de la competencia digital sobre la planificación curricular. Se tiene como resultados que el Chi-2 es de 29,770, así como el nivel de significancia de 0.000 menor a 0.05 , lo que indica que dichos datos se ajustan al modelo, y en consecuencia, se rechaza la hipótesis nula y se concluye que la competencia digital docente influye significativamente en la planificación curricular.

Tabla 6. Pseudo R cuadrado

\begin{tabular}{ll}
\hline Cox y Snell &, 194 \\
Nagelkerke &, 403 \\
McFadden &, 329 \\
\hline
\end{tabular}

Función de enlace: Logit.

Interpretación: En la prueba Pseudo R2, se evidencia la dependencia porcentual de la planificación curricular de los docentes de los CEBA de ciclo avanzado de la DRE Ica, el coeficiente de Cox y Snell es de 0.194 y el coeficiente de Nagelkerke de 0.403, el cual indica que la variabilidad de la planificación curricular se debe al $19.4 \%$ y $40.3 \%$ de la competencia digital respectivamente.

\section{Discusión}

De los hallazgos referentes al objetivo general que consistió en determinar la influencia de la competencia digital en la planificación curricular, se pudo concluir que la competencia digital de los docentes provoca una variación significativa en la planificación curricular, aquellos datos fueron corroborados por el valor del coeficiente de Cox y Snell y Nagelkerke, en consecuencia, se rechazó la hipótesis nula.

Dichos resultados guardan similitud con los obtenidos por Plua (2020), en su estudio de nivel correlacional entre competencia digital y comunicación interna, concluyendo que se necesita de la competencia digital, apego y afiliación a la escuela donde labora para realizar un trabajo conjunto, fundamental para el cumplimiento de metas institucionales. Además, son antagónicos con los de Avellán (2019), ya que su estudio tuvo una significancia superior al margen de error, por tal motivo no era necesario evidenciar el grado de influencia, concluyendo que si las competencias digitales de los docentes tienen un nivel alto necesariamente no provoca variación alguna en la administración de las escuelas. 
Al respecto, Almerich et ál. (2018), mencionó que la competencia digital es un cúmulo de destrezas, habilidades y conocimientos que son adquiridos por los sujetos (docentes), los cuales facultan el manejo de diversas herramientas tecnológicas, además, Beneyto y Collet-Sabé (2018), manifestaron que el mayor inconveniente no es el uso de herramientas tecnológicos sino que estas no tengan efecto en la práctica docente, en tal sentido, se puede deducir que la competencia digital va de la mano de la buena planificación curricular, ya que los propósitos que nos planteemos serán enriquecidos por la diversidad de herramientas tecnológicas que encontremos y de nuestra habilidad de poder utilizarlas con pertinencia con el objetivo de brindar una educación de calidad.

\section{CONCLUSIÓN O CONSIDERACIONES FINALES}

- El nivel de competencia digital percibida por los docentes de los CEBA de ciclo Avanzado del departamento de Ica, evidencia que el 28,3\% (39) coincidieron que el desarrollo de la competencia digital es intermedio, y que el 71,7\% (99) que es avanzado, asimismo, respecto a la planificación curricular, el 10.1\% (14), de los docentes manifestó que el nivel del mismo es medio y que el 89,9 \% (124) que se encuentra altamente desarrollado.

- Los resultados mostraron la dependencia de la planificación curricular respecto a la competencia digital, determinando que los datos se ajustan al modelo, por tanto, se comprobó la existencia de dependencia de las variables. En efecto, el valor de Cox y Snell resultó 0.194 y de Nagelkerke de 0.403, lo cual significa que existió un 19.4\% y $40.3 \%$ respectivamente de incidencia de la competencia digital en la planificación curricular.

\section{LISTA DE REFERENCIAS}

Almerich, G., Díaz-García, I., Cebrián-Cifuentes, S. y Suaréz-Rodriguez, J. (2018) Estructura dimensional de las competencias del siglo XXI en alumnado universitario de educación. RELIEVE, 24(1), 1-21. https://doi.org/10.7203/relieve.24.1.12548

Arriaga, M. (2016). El diagnóstico educativo, una importante herramienta para elevar la calidad de la educación en manos de los docentes. Revista Científico Pedagógica Atenas, 3(31), 63-74. https://www.redalyc.org/pdf/4780/478047207007.pdf

Avellán (2019). Las competencias digitales en los docentes y su influencia en la administración escolar de la Unidad Educativa Nueva Aurora - Ecuador, 2019. 
Pineda Morán y otros...

[Tesis de maestría, Universidad César Vallejo]. https://hdl.handle.net/20.500.12692/42732

Aza, P., y Lica, J. (2019). Planificación curricular de los docentes y la cultura ambiental en los estudiantes de educación básica. Revista Innova Educación, 1(2), 172-183. http://www.revistainnovaeducacion.com/index.php/rie/article/view/25/45

Beneyto-Seonane, M., y Collet-Sabé, J. (2018). Análisis de la actual formación docente en competencias TIC. Por una nueva perspectiva basada en las competencias, las experiencias y los conocimientos previos de los docentes. Revista de Curriculum y Formación del Profesorado, 22(4), 91-110. https://recyt.fecyt.es/index.php/profesorado/article/view/69413

Carhuancho, I. M., Nolazco, F. A., Sicheri, L., Guerrero, M. A., y Casana, K. M. (2019). Metodología para la investigación holística. (1ra. Edición). UIDE. https://repositorio.uide.edu.ec/bitstream/37000/3893/3/Metodolog\%c3\%ada\%20 para\%20la\%20investigaci\%c3\%b3n\%20hol\%c3\%adstica.pdf

Carrasco, S (2017). Metodología de la investigación científica. Perú. San Marcos

Donoso-Díaz, S., y Benavides-Moreno, N. (2018). Prácticas de gestión de los equipos directivos de escuelas públicas chilenas. Revista Brasileira de Educação, 23, 128. http://dx.doi.org/10.1590/S1413-24782018230013

Flores, G. (2006). Planificación y programación curricular en la educación física. Universidad Nacional de Educación Enrique Guzmán y Valle. https://nanopdf.com/download/procesos-de-la-planificacion-curricular_pdf\#

García, A., Muñoz-Repiso, V., y Martin del Pozo (2016). Análisis de las competencias digitales de los graduados en titulación de maestros. Revista Latinoamericana de tecnología educativa, 15(2), 155-168. https://core.ac.uk/download/pdf/94142698.pdf

Hernández, R. y Mendoza, C. (2018). Metodología de la investigación. Las rutas cuantitativas, cualitativas y mixtas. Mc. Graw Hill Education. http://virtual.cuautitlan.unam.mx/rudics/wpcontent/uploads/2019/02/RUDICSv9n18p92_95.pdf

Idrovo-Ortiz, F. X. (2019). Las competencias digitales. Una propuesta de integración con el ciclo de aprendizaje. Revista científica Dominio de las Ciencias, 5(1), 431-450. http://dx.doi.org/10.23857/dc.v5i1.1053 
INTEF. Instituto Nacional de Tecnologías Educativas y de Formación del Profesorado. España: Ministerio de Educación, Cultura y Deporte (2017). Marco Común de Competencia Digital Docente. https://aprende.intef.es/sites/default/files/201805/2017_1020_Marco-Com\%C3\%BAn-de-Competencia-Digital-Docente.pdf

Lévano-Francia, L., Sánchez, S., Guillén-Aparicio, P. Tello-Cabello, S., Herrera Paico, N., Collantes-Inga, Z. (2019). Competencias digitales y educación. Propósitos y Representaciones, 7(2), 569-588. http://dx.doi.org/10.20511/pyr2019.v7n2.329

Lozano, J. (2018). Cómo realizar la programación didáctica en formación profesional. Editorial Síntesis S.A.

Martínez-Garcés, J. (2020). Competencias digitales y el reto de la educación virtual derivado de la Covid- 19. Corporación Universitaria Autónoma de Nariño, Santiago de Cali. Colombia. Revista Educación y Humanismo, 22(39), 1-16. https://doi.org/10.17081/eduhum.22.39.4114

Marza, M., y Cruz, E. (2018). Gaming como Instrumento Educativo para una Educación en competencias Digitales desde los Academic Skills Centres. Revista General de $\begin{array}{llll}\text { Información } & y & \text { Documentación, } & \text { 28(1), }\end{array}$ http://dx.doi.org/10.5209/RGID.60805

MINEDU. (2016). Resolución de secretaria General $N^{\circ} 505$ - 2016. Estrategia nacional de las tecnologías digitales en la educación básica, 2016 -2021, de las Tic a la inteligencia digital. https://www.gob.pe/institucion/minedu/normaslegales/124210-505-2016-minedu

MINEDU. (2017a). Cómo planificar el proceso de enseñanza, aprendizaje y evaluación formativa. Lima. Recuperado de www.minedu.gob.pe/curr

MINEDU. (2017b). Currículo nacional. Lima: Fondo Editorial del Minedu. http://www.minedu.gob.pe/curriculo/pdf/curriculo-nacional-de-la-educacionbasica.pdf

MINEDU. (2020). R.M. $N^{\circ} 160-2020-M I N E D U$. Disponen el inicio del año escolar a través de la implementación de la estrategia denominada "Aprendo en casa”, a partir del 6 de abril de 2020 y aprueban otras disposiciones. https://www.gob.pe/institucion/minedu/normas-legales/466108-160-2020minedu

Navarro, Y., Pereira, M., de Homes, L. P., y Cascioli, N. F. (2010). Una mirada a la 
planificación estratégica curricular. Telos: Revista de estudios interdisciplinarios en Ciencias $\quad$ Sociales, $\quad 12(2), \quad 202-216$. https://dialnet.unirioja.es/servlet/articulo?codigo=3335360

Navarro, D., y Samón, M. (2017). Redefinición de los conceptos método de enseñanza y método de aprendizaje. EduSol, 17(60), 26-33. https://www.redalyc.org/jatsRepo/4757/475753184013/html/index.html

Plua, M. S. (2020). Competencia digital y comunicación interna en los docentes de la Unidad Educativa Violeta Luna Carrera, Ecuador 2019. [Tesis de maestría, Universidad César Vallejo]. https://hdl.handle.net/20.500.12692/43417

Pompa, Y. C., y Pérez, I. A. (2015). La competencia comunicativa en la labor pedagógica. $\begin{array}{llll}\text { Revista Universidad } \quad y \quad \text { Sociedad, } & \text { 160-167. }\end{array}$ http://scielo.sld.cu/scielo.php?script=sci_arttext\&pid=S221836202015000200023

Siemens, G. (2004) Connectivism: a theory for the digital age' eLearningSpace.

UNESCO. (2013). Uso de tic en educación en América Latina y el Caribe. Análisis regional de la integración de las TIC en la educación y de la aptitud digital (ereadiness). Londres: Unesco.

UNESCO. (2020), Educación en crisis, desafíos del camino a seguir. https://es.unesco.org/news/educacion-crisis-desafios-del-camino-seguir

Vargas-Murillo, G. (2019). Competencias digitales y su integración con herramientas tecnológicas en educación superior. Cuadernos Hospital de Clínicas, 60(1), 8894. http://www.scielo.org.bo/pdf/chc/v60n1/v60n1_a13.pdf 